\title{
Modelling of the temperature field that accompanies friction stir welding
}

\author{
Przemysław Nosal $^{1, *}$, and Artur Ganczarski ${ }^{1}$ \\ ${ }^{1}$ Cracow University of Technology, Institute of Applied Mechanics, Jana Pawła II 37, 31-864 Cracow, Poland
}

\begin{abstract}
The thermal modelling of the Friction Stir Welding process allows for better recognition and understanding of phenomena occurring during the joining process of different materials. It is of particular importance considering the possibilities of process technology parameters, optimization and the mechanical properties of the joint. This work demonstrates the numerical modelling of temperature distribution accompanying the process of friction stir welding. The axisymmetric problem described by Fourier's type equation with internal heat source is considered. In order to solve the diffusive initial value problem a fully implicit scheme of the finite difference method is applied. The example under consideration deals with the friction stir welding of a plate ( $0.7 \mathrm{~cm}$ thick) made of Al 6082-T6 by use of a tool made of tungsten alloy, whereas the material subjected to welding was TiC powder. Obtained results confirm both quantitatively and qualitatively experimental observations that the superior temperature corresponds to the zone where the pin joints the shoulder.
\end{abstract}

\section{Introduction}

At the end of the XX century Wayne Thomas and his team in The Welding Institute in Cambridge UK (TWI) developed and patented new technology of the materials joined by the friction with the mixing of plastic material in the welding zone. This technology is known as Friction Stir Welding (FSW) [1, 2]. FSW is a steady state process, which means that the material does not reach melting point and that it brings some advantages in the form of a defect free joint, which is known from traditional welding techniques $[2,3]$. Frictional heat is generated by the friction between the tool and the base material to soften the material in the joining zone. Moreover, the plastic work of the material supplies additional portions of heat energy [4]. The mechanical influence of the tool leads to grain fragmentation inside the weld that has a positive effect on the mechanical properties of the joint. That is the reason why joints obtained by the use of FSW technology have better parameters than joints produced by more traditional methods e.g. MIG welding method [3]. FSW technology allows the joining of materials which are regarded as difficult to weld and materials which are dissimilar e.g. aluminium with copper [3]. At present, few countries around the world are developing this technology and are implementing it in various branches of industry like automotive, railway, aeronautic, ship-building and space industry [4]. Friction stir welding, besides its undeniable benefits in joining areas of different kinds of materials, has also other interesting applications. One of them is the possibility to manufacture composites via mixing one material phase, e.g. in the form of powder, in the other one which is a matrix (Fig.1). The received material manifests certain attributes of the Functionally Graded Materials (FGM) material.

Friction Stir Processing (FSP) is a variant of friction stir welding technology that includes all unique and beneficial features of this joining method [5-9]. FSP process allows us to change the properties of modified material through the creation of fine grained structures [5], surface composites [6-9] and modifications of cast alloys microstructure. One of the very few examples is research by Gandra [8], who observed that composite of AA5083 alloy and introduced $\mathrm{SiC}$ particles exhibit orthogonal gradients of composition and mechanical behaviour.

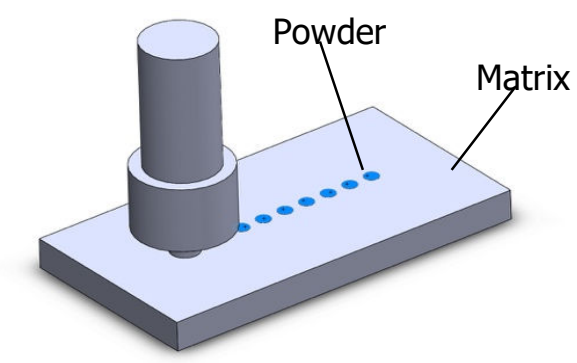

Fig. 1. TiC powder implementation approach onto the aluminium plate in the FSP process.

Liu et al. [9] observed that the best properties of reinforced aluminium matrix composites fabricated by FSP process are received after a few passes of the welding tool. The FSW process is associated with significant growth of the joining materials enthalpy,

\footnotetext{
*Corresponding author: nosal.przemyslaw@student.pk.edu.pl
} 
which allows their plastic strain and the adhesion phenomenon process. Moreover, the high temperature initiates the diffusion processes in materials that have different physical and chemical properties.

\section{Mathematical model}

Material flow around the mixing pin is a very complex phenomenon and it is determined by the specific geometry of the tool. Additionally, during the exact welding process compound of the direction of the rotary motion with linear motion of the FSW tool leads to asymmetric material flow in welding the zone, which causes asymmetry in the joint construction (Fig.2).

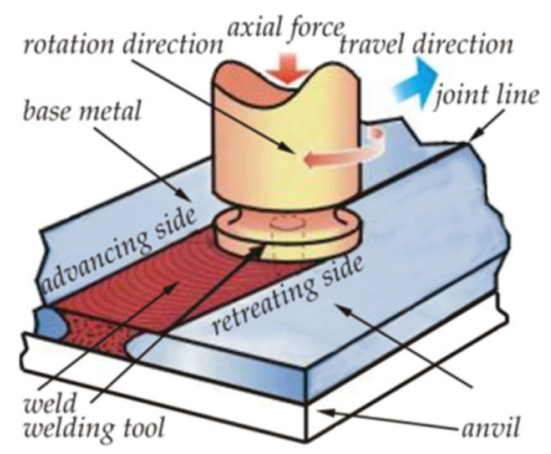

Fig. 2. Scheme of the FSW process [10].

With respect to this fact, we can distinguish two sides in the FSW joint: advancing side and retreating side. They depend on the relations between rotary and linear motion of the welding tool.

a)

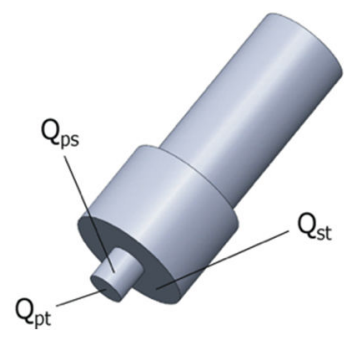

b)

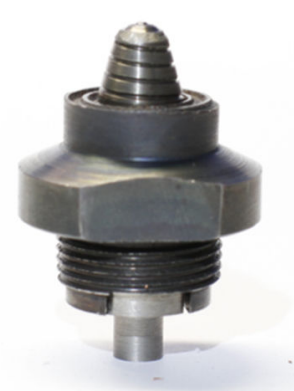

Fig. 3. FSW tool: a) respective tool areas that determine amount of generated heat energy, b) real welding tool with conical pin and cut spirals on the pin and the shoulder.

This article focuses only on the pure thermal issue of the first stage of the FSW process, namely the stage when the welding tool starts penetrating the work piece.
Thermal modelling is the first step to predict the possible occurrence of voids or defects in the joint as well as microstructure evolution and finally properties of the joint [6]. All the processes are dependent on the heat that is generated during welding; therefore, thermal history has a large impact on the quality of the joint. Two basic components of the welding tool are the shoulder and the pin (Fig.3). Their basic function is generating heat energy by the friction between materials of the tool and the work piece. The second function is mixing of the plastic material inside the welding zone. During the FSW process, mechanical energy is transformed into heat energy. The fundamental assumption made by the whole energy that is generated during welding process is its division into the parts that are described respectively by active areas of the welding tool with the cylindrical pin. This energy can be described by the equation $[10,11]$

$$
Q_{T}=Q_{p s}+Q_{p t}+Q_{s t}
$$

Where the individual members respond to the heat which is generated on the $\operatorname{pin} \operatorname{side} Q_{p s}, \operatorname{pin} \operatorname{tip} Q_{p t}$, shoulder tip $Q_{s t}$ and these components are described by the following integrals

$$
\left.\begin{array}{l}
Q_{p s}=\int_{0}^{2 \pi} \int_{0}^{h} \omega \tau_{\text {cont }} r^{2} d \varphi d z \\
Q_{p t}=\int_{0}^{\frac{d}{2}} \int_{0}^{2 \pi} \omega \tau_{\text {cont }} r^{2} d \varphi d r \\
Q_{s t}=\int_{0}^{2 \pi} \int_{\frac{d}{2}}^{\frac{D}{2}} \omega \tau_{\text {cont }} r^{2} d \varphi d r
\end{array}\right\}
$$

Most of the welding tools have threads on the probe, which increase the effective friction coefficient and the frictional heat, which is generated by the pin [12]. Friction phenomenon occurring during FSW process is very difficult to model because welded material can stick to the material of the tool or can slip. Furthermore, it is non-linear and the value of friction coefficient is not constant. Between these two materials places where the material was melted may occur [6]. The basic equation for the heat generation at the contact surface is [2]

$$
\dot{q}_{V}=\tau_{\text {cont }} \omega r
$$

Where $\tau_{\text {cont }}$ is the contact shear stress of the interface located between shear layer of workpiece and the tool, $\omega$ is a radial frequency and $r$ is a radius of the tool. The next simplification deals with the spatial dispersion of the volume intensity heat source assumed in form of (compare [13])

$$
\dot{q}_{V}=\frac{3 Q_{T} r}{2 \pi R^{3} w_{s l}}
$$

This is the extended version of Eq. (3) and manifests itself as excitation in the rotational symmetry and nonstationary Fourier equation

$$
\lambda\left(\frac{\partial^{2} \theta}{\partial r^{2}}+\frac{1}{r} \frac{\partial \theta}{\partial r}+\frac{\partial^{2} \theta}{\partial z^{2}}\right)+\dot{q}_{V}=\rho c_{V} \frac{\partial \theta}{\partial t}
$$

Variable $w_{s l}$ means width of shear layer (Fig.4). In reality, the heat source is heterogeneous and it is closely connected with respective active surface, 
which nonlinearly affects the work piece, because the tool during the first stage of welding is penetrating material and the heat energy is lower than the heat energy at the end of this stage. In general, heat energy generated by the pin surface is negligible when compare to the heat energy generated by the shoulder so the energy $Q_{T}$ in (4) can be substituted by $Q_{s t}$, in thick plate $Q_{\text {pin }} \cong 10 \% Q_{T}$ [6]. Mijajlovic and Milcic [10] presented analytical model for estimating the amount of heat generated during FSW, which is a process of mechanical energy transformation and considers many parameters such as: tool rotation speed, angular velocity, travel rate, tilt angle, axial force, torque, etc. Additionally, they estimated values of parameters which are mutually dependent and which influence the heat generation process.

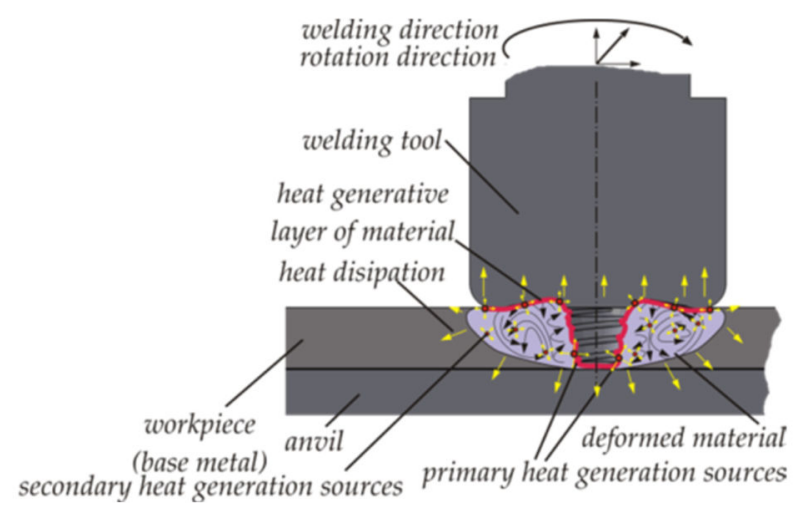

Fig. 4. Scheme of heat generation during FSW process [10].

In order to reach the unique solution of the Eq. (5) the following boundary conditions can be applied: $\theta_{\infty}=20^{\circ} \mathrm{C}$, adiabatic condition on the symmetric axis and the third order boundary conditions, which are modelling free convection on the extant boundaries of the showed area on the Fig. 5 .

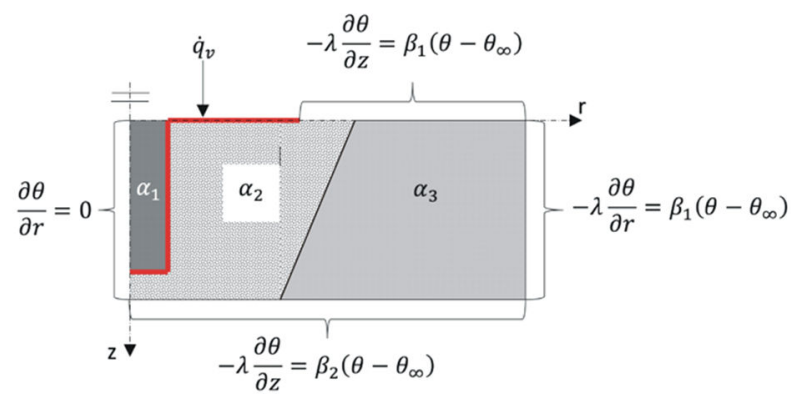

Fig. 5. Geometrical scheme and the boundary conditions of the analysed problem (von Neumann's stability coefficients for: tool- $\alpha_{1}$, added phase $-\alpha_{2}$ and matrix $-\alpha_{3}$, respectively)

$2 \mathrm{D}$ model is subject to validation and experimental verification. As the next stage, this model can be developed to a 3D model with heat generated by friction like in [14] and plastic work of material. Shi et al. [15] presented the model of heat transfer and material flow in Reverse Dual-Rotation FSW process by using CFD simulations. Similarly, Micallef et al. [16] used a thermo- fluid-flow model in computational fluid dynamics simulation to predict material flow and heat gradients. The coupled thermal flow model was also developed by Hamilton [17] to simulate FSP process by use of commercial software. Two different methods/models for rotating tools are presented by Kang et al. [18]. They assumed heat generation only by the plastic deformation not by friction. Finite volume method with Eulerian's description approach was adopted. First, the model assumes that only the contact area rotates and the plastic zone grows as heat spreads. Secondly, the model provides a plastic deformation zone named the rotation affected zone (RAZ), as a place where heat energy is generated. In work [19] Song and Kovacevic submitted equation for heat energy which is generated by the threaded surface of the pin.

$$
Q_{p i n}=2 \pi r_{p} h k \bar{Y} \frac{V_{m}}{\sqrt{3}}+\frac{2 \mu k \bar{Y} \pi r_{p} h V_{r p}}{\sqrt{3\left(1+\mu^{2}\right)}}+\frac{4 F_{p} \mu V_{m} \cos \varphi}{\pi}
$$

where $\varphi=90^{\circ}-\psi-\tan ^{-1}(\mu), V_{m}=\frac{\sin \psi}{\sin \left(180^{\circ}-\varphi-\psi\right)} v_{p}$, $V_{r p}=\frac{\sin \varphi}{\sin \left(180^{\circ}-\varphi-\psi\right)} v_{p}, v_{p}=r_{p} \omega \cdot r_{p}$ - radius of the pin, $h$ - the thickness of the work piece, $\psi$ - an angle of the threads helix, $F_{p}$ - the translation force during the FSW process and $\bar{Y}$ - the average shear stress of the welded material. Attallah [11] presented alternating direction implicit (ADI) scheme to solve unsteady heat transfer equation by finite difference method. Stability of ADI scheme is only restricted to being non-oscillating and finite. Time increment was described by equation

$$
\Delta t<\frac{(\Delta x)^{2}+(\Delta y)^{2}+(\Delta z)^{2}}{8 \alpha_{\max }}
$$

Appropriate value of time step allows accuracy and convergence of simulation.

\section{Example}

The simulation of the first stage of FSP process was made experimentally. The rotating welding tool penetrated the work piece with constant feed-rate and after achieving the set depth it was dwelling for a few seconds (Fig. 6). The workpiece and the matrix were made from aluminium plate and the second phase was titanium carbide powder.

Table 1. Physical properties of the tool material, TiC powder and the aluminium plate.

\begin{tabular}{|l|c|c|c|}
\hline \multicolumn{1}{|c|}{ Material } & $\left.\rho \mathrm{g} / \mathrm{cm}^{2}\right]$ & $\lambda[\mathrm{W} / \mathrm{cm} \cdot \mathrm{K}]$ & $c_{\mathrm{v}}[\mathrm{J} / \mathrm{g} \cdot \mathrm{K}]$ \\
\hline $\begin{array}{l}\text { Tool -Tungsten } \\
\text { alloy }\end{array}$ & 19.25 & 175 & 0.135 \\
\hline $\begin{array}{l}\text { Added phase -TiC } \\
\text { powder }\end{array}$ & 4.92 & 110 & 0.520 \\
\hline Matrix Al 6082-T6 & 2.71 & 172 & 0.894 \\
\hline
\end{tabular}


Comparison of the basic physical properties of materials used is shown in Tab. 1. Solution of the boundary problem (5) is achieved by using finite difference method based on the fully implicit scheme [20]

$$
\begin{gathered}
\alpha\left(1-\frac{\Delta r}{2 r_{i}}\right) \theta_{i-1, j}^{\text {new }}+\alpha \theta_{i, j-1}^{\text {new }}-(1+4 \alpha) \theta_{i, j}^{\text {new }}+ \\
\alpha \theta_{i, j+1}^{\text {new }}+\alpha\left(1+\frac{\Delta r}{2 r_{i}}\right) \theta_{i+1, j}^{\text {new }}=-\theta_{i, j}^{\text {old }}-\frac{\dot{q}_{V} \Delta t}{\rho c_{V}}
\end{gathered}
$$

Where the symbol $\alpha=\frac{\lambda}{\rho c_{V}} \frac{\Delta t}{(\Delta r)^{2}}$ denotes von Neumann's stability coefficient, the magnitude of each differs for subsequent three materials. The differential scheme (8) is applied to each point of mesh at a size $35 \times 90$ that discretizes rectangle domain. Magnitudes of unknown sat points located outside the domain are expressed by combinations of unknown points by use of appropriate boundary conditions (see Fig. 5). Finally, the set linear algebraic equations, which exhibit a structure typical for sparse systems, are solved by use of the conjugate gradient method and the row-indexed compact storage [20].

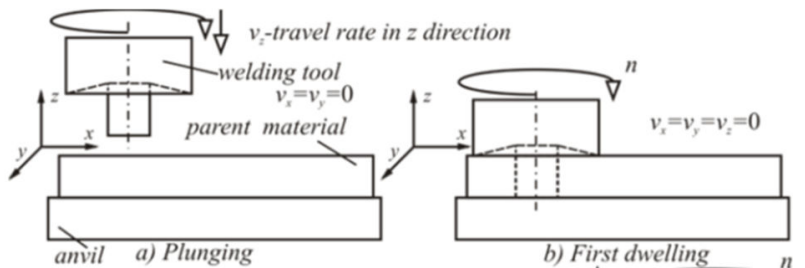

Fig. 6. Scheme of FSW/FSP first stage [10].

Obtained results involving the evolution of the temperature field shown in Fig. 7, confirm quantitatively and qualitatively temperature distribution field as well as theoretical results published by others scientists and empirical results reach by experimental tests. The presented model predicts achievement of the maximal temperature near to $310^{\circ} \mathrm{C}$ in location where the tool pin and shoulder meets. The temperature field is homogeneous in the thermo-mechanical affected zone (TMAZ). The temperature drops from $300^{\circ} \mathrm{C}$ to $270^{\circ} \mathrm{C}$ outside this zone of what is typical for heat-affected zone (HAZ). Individual zones in produced composite respond to the zones that are known from FSW joint (Fig. 8). Their heterogeneous areas are correlated with the obtained results from numeric simulation.

a)
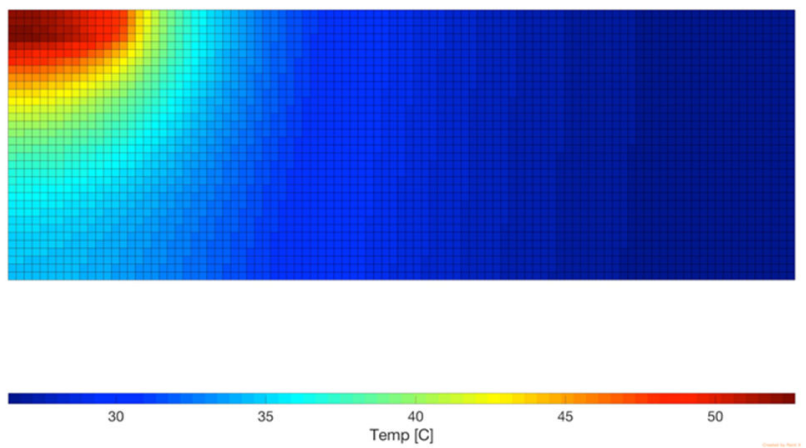

b)
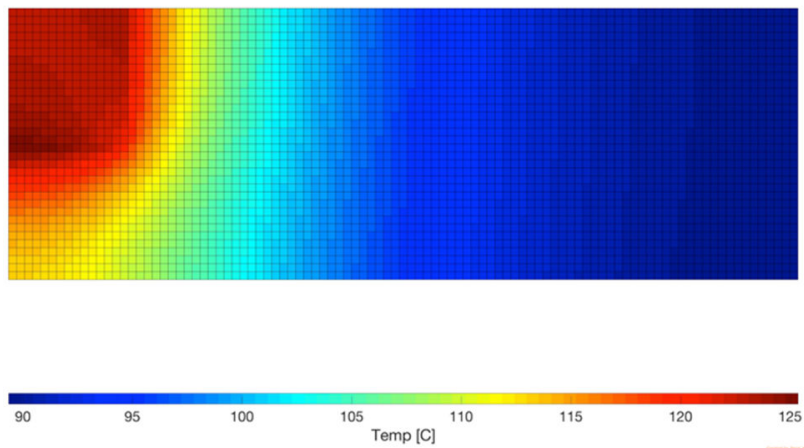

c)
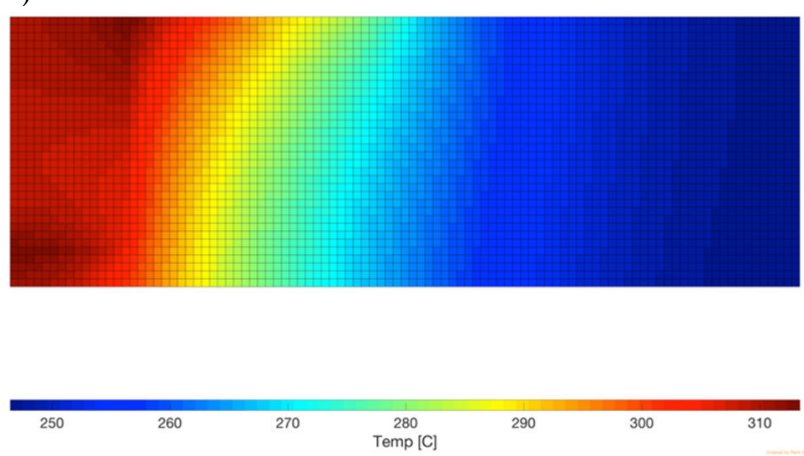

Fig. 7. Temperature fields in different period of the tool penetration in the base material: a) time $4 \mathrm{~s}-$ depth of the pin $0.8 \mathrm{~mm}, \mathrm{~b})$ time $18 \mathrm{~s}-$ depth $3.6 \mathrm{~mm}, \mathrm{c}$ ) time $33 \mathrm{~s}-$ depth 6 $\mathrm{mm}$.

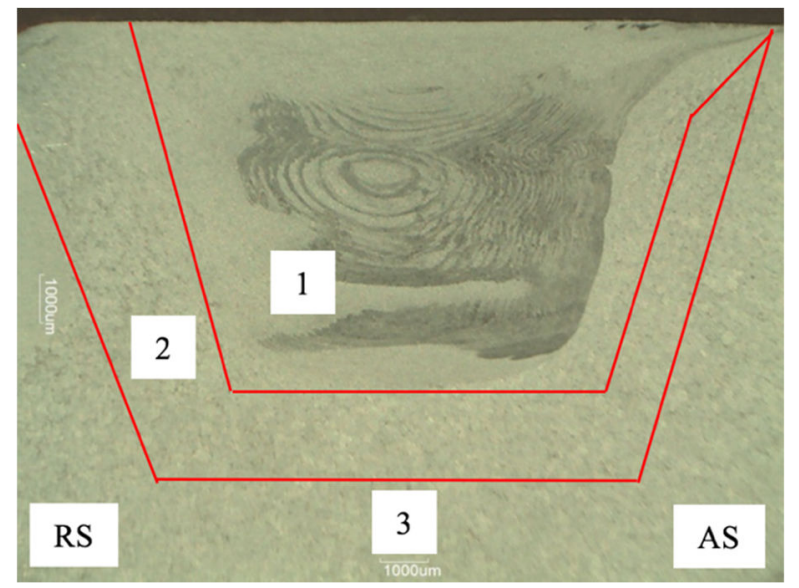

Fig. 8. Macrostructure image of sample Al-TiC composite produced with one pass of welding tool: AS - advancing side, $\mathrm{RS}$ - retreating side, 1 - weld nugget, 2 - thermo-mechanically affected zone, 3 - heat affected zone.

Observed black curves in the weld are introduced by $\mathrm{TiC}$ powder. An incomplete mix of $\mathrm{TiC}$ powder in to the aluminium matrix is visible as a result of one passing of the welding tool.

\section{Conclusions}

The following concluding remarks may be formulated for obtained results:

- the maximal temperature is lower than the melting temperature of aluminium which corresponds to FSP process property - solid state joining process, 
- the highest temperature occurs in place where the pin and shoulder meet, which corresponds with the place where generated heat has no possibility of dissipation,

- the occurrence of the highest temperature was observed around 33 seconds at the end of the first stage of FSW process,

- isotherm dispersion outside the TMAZ area corresponds to the HAZ area observed in the photos from the metallurgical test.

Further research will focus on a mathematical model that would include mechanical influences on heat energy generation. Additionally, there will be an attempt to analytically predict some of the parameters (e.g. axial force, torque) which have large impact on the amount of generated energy but cannot be measured without specialized equipment.

The author is indebted to Dr Marek Hebda for help in metallography research and valuable clues. Presented sample of FSP composite was produced in Electriscompany, ELECTRIS, Mickiewicza 6, 32-650 Kęty, Poland.

\section{References}

1. W.M. Thomas, E.D. Nicholas, J.C. Needham, M.G. Murch, P. Temple-Smith, C.J. Dawes,Improvements relating to friction stir welding, US Patent No 5460317, 1999

2. D. Lohwasser, Z. Chen, Friction Stir Welding. From basics to applications (CRC, Cambridge, 2010)

3. P. Nosal, Mechanical properties of friction stir welded copper plates joints(AGH, Kraków, 2016)

4. A. Ambroziak, Friction welding of materials with various properties (OW PolitechnikiWrocławskiej, Wrocław, 2011)

5. R.S. Mishra, M.W. Mahoney, Friction Stir Welding and Processing (ASM International, Ohio, 2008)
6. M.S. Węglowski, S. Dymek, C.B. Hamilton, Bulletin Of The Polish Academy of Sciences Technical Sciences 61, 4 (2013)

7. Y.X. Gan, D. Solomon, M. Reinbolt, Materials3,329-350 (2010)

8. J. Gandra, R. Miranda, P. Vilaca, A. Velhinho, J. Pamies Teixeira, Jour. of Mat. Proce. Tech.211, 1659-1668 (2011)

9. Q. Liu, L. Ke, F. Liu, C. Huang, L. Xing, Mate. and Design45, 343-348 (2013)

10. M. Mijajlowic, D. Milcic, Chap. 11, InTech, 2012

11. M.M. Attallah, Friction stir welding of aluminium alloys. Microstructure-property development (LAP, Birmingham, 2007)

12. M. Schwartz, Innovations in Materials Manufacturing, Fabrication and Environmental Safety (CRC, New York, 2011)

13. Y.J. Chao, X. Qi, Trans. of the ASME 125, 138145 (2003)

14. G.S. Reese, Analytical Thermal Model of Friction Stir Welding with Spatially Distributed Heat Source(BYU Scholar Archive, Brigham, 2012)

15. L. Shi, C.S. Wu, H.J. Liu, JMEPEG23, 2918-2929 (2014)

16. D. Micallef, D. Camilleri, A. Toumpis, A. Galloway, L. Arbaoui,SAGEJou. 230, 1-26 (2015)

17. C. Hamilton, M.S. Węglowski, S. Dymek, MMMS and ASM Inter. 46B, 1409-1418(2015)

18. S.W. Kang, B.S. Jang, J.W. Kim, A study on heatflow analysis of friction stir welding on a rotation affected zone, JMST 28, 3873-3883 (2014)

19. M. Song, R. Kovacevic, IJMTM43,605-615 (2003)

20. W.H. Press, S.A.Teukolsky, W.T. Vetterling, B.P. Flannery, Numerical recipes in fortran(Cambridge University Press, Cambridge, 1993) 